\title{
Entre las transferencias económicas y la \\ privatización: Tendencias de las políticas de inclusión educativa en Argentina y Uruguay
}

Espejo Villar, L. Belén

Universidad de Salamanca, España

Ibev@usal.es

Calvo Álvarez, M. ${ }^{a}$ Isabel

Universidad de Salamanca, España

isabelc@usal.es

\section{Resumen}

El presente trabajo parte de las directrices políticas establecidas por la Unesco en el Objetivo 4 del Desarrollo Sostenible encaminado a conseguir una educación inclusiva, equitativa y de calidad, así como a promover oportunidades de aprendizaje durante toda la vida y para todos.

Si bien las estrategias a las que se hace referencia en el documento y que hemos querido centrar en las políticas y los actores, aparecen en el texto dirigidas a un mayor número de colectivos que en el Objetivo 2 del Desarrollo del Milenio, un estudio en profundidad de la nueva agenda educativa nos permite comprobar que, a pesar de ser más amplios los grupos de interés y las metas con las que se trabaja, las medidas que se contemplan tienen una orientación mayoritariamente asistencial y compensatoria que resultan difícil de entender en este marco.

En este sentido, el énfasis que se proporciona a los programas de segunda oportunidad y a la reinserción, además de la prestación de servicios básicos como los de salud y alimentación, siendo necesarios, indican una descompensación en la gestión de la inclusión, al omitir aspectos que deberían estar más relacionados con la dimensión preventiva y con el trabajo que deben realizar las instituciones educativas en la consecución de logros de aprendizaje del colectivo que no ha abandonado el sistema educativo.

Tomando como punto de partida los programas de transferencias de efectivo, en tanto que medida política y también centrados en el sector privado (como actor), examinamos las tendencias que están desarrollando Argentina y Uruguay en materia de políticas de inclusión. La elección de los países está motivada por ser dos de los tres estados, junto con Chile, del cono sur de América Latina que diseñaron medidas específicas de inclusión a finales de los 90 y que con algunas particularidades comenzaron a implementarse en la segunda mitad de la década del 2000.

Por tanto, analizamos la línea de apoyo al sector privado que Uruguay ha adoptado desde hace unos años para favorecer la inclusión en la educación secundaria y que es compartida por Argentina en la privatización de las medidas inclusivas.

\section{Abstract}

This work is based on the political guidelines established by Unesco in Goal 4 of Sustainable Development aimed at achieving an inclusive, equitable and quality education, promoting learning opportunities throughout life and for all. Although the strategies referred to in the document that we wished to focus on policies and actors appear in the text aimed at more groups than in Millennium Development Goal 2, an in-depth study of the new educational agenda allows us to verify that, even though the interest groups and the goals to be worked upon are broader, the measures contemplated are mainly oriented towards assistance and compensation, something difficult to understand in this framework.

In this regard, the emphasis that is given to second chance programs and reintegration, in addition to the provision of basic services such as health and nutrition, while necessary, point to an imbalance in the management of inclusion, omitting aspects that should be more oriented towards prevention and the work that educational institutions must carry out to achieve the learning goals of those who have not left the educational system.

Taking as a starting point the cash transfer programs, as a policy measure and also focusing on the private sector (as an actor), we examine the trends that Argentina and Uruguay are developing regarding inclusion policies. These countries were chosen because they are two of the three states, together with Chile, in the southern cone of Latin America that designed specific inclusion measures at the end of the $90 \mathrm{~s}$ and that with some particularities began to be implemented in the second half of the 2000 s.

We thus analyse the line of support to the private sector that Uruguay adopted a few years ago to favour inclusion in secondary education and that is shared by Argentina in the privatization of inclusive measures.

Palabras clave: Gestión privada, transferencias económicas, desarrollo asistencial, políticas de inclusión, educación secundaria, gobernanza.

Keywords: Private management, economic transfers, social welfare policy, inclusion policies, secondary education, governance. 


\section{INTRODUCCIÓN}

La aprobación del Objetivo de Desarrollo Sostenible 4 ha supuesto un reconocimiento a escala mundial por la interpretación comprometida y novedosa que las instituciones hacen, a priori, de la educación. No cabe duda de que plantear la construcción del modelo educativo desde principios que abanderan paradigmas contrapuestos en políticas socioeducativas representa una responsabilidad gubernamental extraordinariamente ambiciosa. De modo que, tratar de garantizar una educación inclusiva y equitativa de calidad y promover oportunidades de aprendizaje para todos, constituye el primer paso en el ejercicio de desbloquear fronteras ideológicas que han sido sustentadas históricamente por el discurso de la calidad (definido en ámbitos jurídicos conservadores en términos de exclusión-selección), así como por la defensa de la equidad, llevada en la agenda 2030 a su forma más radical de igualdad y trazada de acuerdo con premisas de universalidad en el aprendizaje, que ponen en relación valores como la inclusión y el éxito, considerados durante mucho tiempo excluyentes.

En ese sentido los documentos institucionales que se han ido aprobando representan un avance, en tanto que permiten someter a debate las tensiones que se derivan de los ajustes entre las tres macrometas (calidad, cobertura y equidad) cada vez más consensuadas por la comunidad internacional (Bogliaccini, 2018).

Sin embargo, algunos de los acuerdos que formulan los gobiernos en el marco de la Declaración de Incheon (2015) se enfrentan en su concreción estratégica a omisiones y contradicciones que más que buscar la convergencia, parecen establecer vías bidireccionales que anulan cualquier intento de conseguir una igualdad real. Hablamos por ejemplo de que focalizar la reducción de la desigualdad a la eliminación de los obstáculos financieros a través de programas de transferencia de efectivo, de servicios de nutrición y salud, de comidas en las escuelas y/o de programas de segunda oportunidad y reinserción no deja de ser un desplazamiento de las políticas de prevención a los circuitos del asistencialismo, para convertirse en definitiva en una apuesta por la reproducción de sistemas compensatorios.

En este trabajo partimos del interés que está mostrando la agenda política (ODS 4) en construir la estrategia de equidad, inclusión e igualdad de género en base a un enfoque que además de ignorar preocupaciones estructurales y responsabilidades de los Estados en el mantenimiento de la desigualdad, paradójicamente fomenta un sistema de ayuda social subsidiario, al tiempo que refuerza la presencia de mecanismos de gobernanza y opta por sistemas de gestión privada (Martens, 2017; Westheimer, 2015; Wulff, 2017). Y trasladamos este análisis a dos países, Argentina y Uruguay que destacan tanto por su representatividad en cobertura de transferencias monetarias, en el caso de Argentina (Hornes, 2014) como por la creciente tendencia de las alianzas privadas en políticas de inclusión educativa en Uruguay.

\section{TRANSFERENCIAS ECONÓMICAS Y LOS DILEMAS DE LAS POLÍTICAS DE INCLUSIÓN}

¿Son las transferencias económicas la solución estratégica que plantea la Agenda 2030 para alcanzar el ODS 4 o representa más bien una dificultad añadida en el camino trazado hacia la inclusión educativa?

En general, se puede decir que los programas de transferencias condicionadas forman parte del conjunto de políticas sociales que bajo el influjo de organismos internacionales han tratado de reducir la pobreza y luchar contra la desigualdad en el contexto latinoamericano a partir de la década de los 90. El modelo de protección social sobre el que se construyen estos mecanismos monetarios gira en torno a principios de compensación, focalización, eficacia y descentralización (Hornes, 2014), pilares que han ido modelando el papel del Estado hacia un rol de subsidiariedad en escenarios de extrema pobreza, pobreza y vulnerabilidad.

Sin embargo, el desarrollo de este modo de orientar las políticas sociales ha estado sujeto a un debate muy crítico e inconcluso acerca de la conveniencia de su implementación. Frente a la supremacía (defendida por el sector institucional) de las transferencias monetarias en la creación de bienestar social, así como en la modernización de las políticas públicas y en el «fortalecimiento de la institucionalidad social» (Cecchini y Atuesta, 2017, p. 10), otros autores destacan las trampas que supone este tipo de dependencia económica, en la medida que no hacen cumplir las condicionalidades que los programas conllevan, ni impactan en la pobreza, y por tanto no consiguen los objetivos para los que se proponen. 
Más allá de reducir la legitimidad de estos programas al enfrentamiento de los discursos, creemos destacable la idea de que tras la articulación de estas políticas de redistribución subyace el poder que asume el Estado en la institución de garantías fundamentales. Su función no se limita a controlar el uso de la condicionalidad por parte de las familias, sino que se trata de establecer un ejercicio de corresponsabilidad que implica el deber de los poderes públicos de sufragar las transferencias, pero también de garantizar la prestación de los servicios educativos y sanitarios (Baraibar, 2011; Ibarrarán y Cueva, 2017).

En cualquier caso y dada la centralidad con la que estos programas son planteados en la agenda política en tanto que herramienta clave en la consecución de la equidad, conviene recordar que las mejoras en términos de asistencia y permanencia que procuran estos programas no siempre se ven acompañados de un incremento de la calidad educativa, lo que debilita las posibilidades de impactar en las dimensiones (asequibilidad, accesibilidad, aceptabilidad, adaptabilidad) que encierra el derecho a la educación (Tomasevski, 2004, citado por Naya y Dávila, 2017, p. 22).

\subsection{Evolución de los Programas de transferencias económicas en Argentina y Uruguay}

¿Cuál ha sido la evolución que han seguido los programas de transferencias monetarias en Argentina y Uruguay en materia educativa? ¿Existe una línea de reciprocidad entre los incentivos que proporcionan estos mecanismos y las prioridades educativas?

Argentina y Uruguay son, junto a Brasil y Ecuador, los países que en los últimos años presentan una mayor inversión en Programas de Transferencias Condicionadas, tal y como puede comprobarse en la Figura 1.

Figura 1. Inversión Programas de Transferencias.

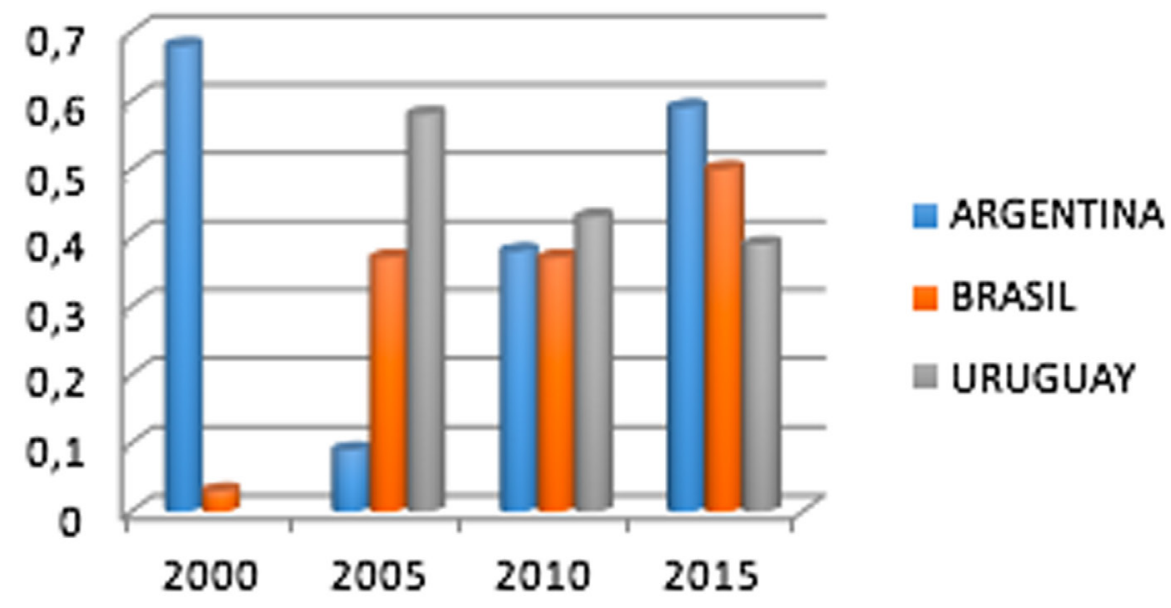

Nota: Elaboración propia adaptado de Cecchini y Atuesta, 2017, CEPAL

Precisamente por ello, y al objeto de establecer una aproximación que nos permita comprender los procesos políticos que subyacen en las actuaciones monetarias de carácter inclusivo, hemos analizado en perspectiva comparada dos iniciativas muy significativas que se desarrollan en el marco de las políticas de inclusión en Argentina y Uruguay.

Estructuralmente, la elección de los programas responde tanto a tendencias de la agenda política 2030 (aprendizaje permanente, programas de segunda oportunidad), como al interés por comprobar los patrones de igualdad en etapas que han tenido menor cobertura educativa, como son la educación superior y la educación terciaria (Ibarrarán, Regalia y Stampini, 2016).

En el caso de Argentina nos hemos centrado en el Programa de Respaldo a Estudiantes Argentinos, conocido como: PROGRESAR, aprobado por el Decreto 84/2014 y modificado por el Decreto 90/2018. 
Se trata de una iniciativa gestada en el marco de una política que pretende dar cumplimiento a la universalidad de la educación secundaria, así como articular trayectorias efectivas para alumnos que cursan esta etapa, como consecuencia de las necesidades derivadas de cambios en los niveles de repetición y abandono escolar en Argentina (CADE, 2018).

En este sentido, se concibe como una actuación compensatoria en el plano económico y en el educativo, dirigida al mantenimiento, pero sobre todo al reingreso formal de jóvenes entre 18 y 24 años (puede llegar hasta los 30 años) que se encuentran en situaciones de vulnerabilidad y que están interesados en terminar estudios obligatorios o en iniciar la educación superior.

La importancia de este programa radica en la institucionalización que se produce del derecho a la educación en el marco de las transferencias monetarias. Sin embargo, y a pesar de su juventud (comienza en el 2014), en los últimos años se están produciendo algunos desajustes por parte de la Administración (anomalías entre instituciones, endurecimiento de requisitos a jóvenes en situaciones de vulnerabilidad...) que podrían desestabilizar compromisos políticos internacionales (Cirmi, 2017). Observando la presencia de alumnos matriculados en el Programa en el periodo comprendido entre julio de 2016 a marzo de 2017 (Figura 2), nos quedan dudas de la existencia de una cultura de corresponsabilidad entre las prioridades políticas y las educativas.

Figura 2. La divergencia entre las lógicas políticas y los intereses educativos en el Programa PROGRESAR. Número de matrículas realizadas.

1.200 .000

1.000 .000

800.000

600.000

400.000

200.000

0
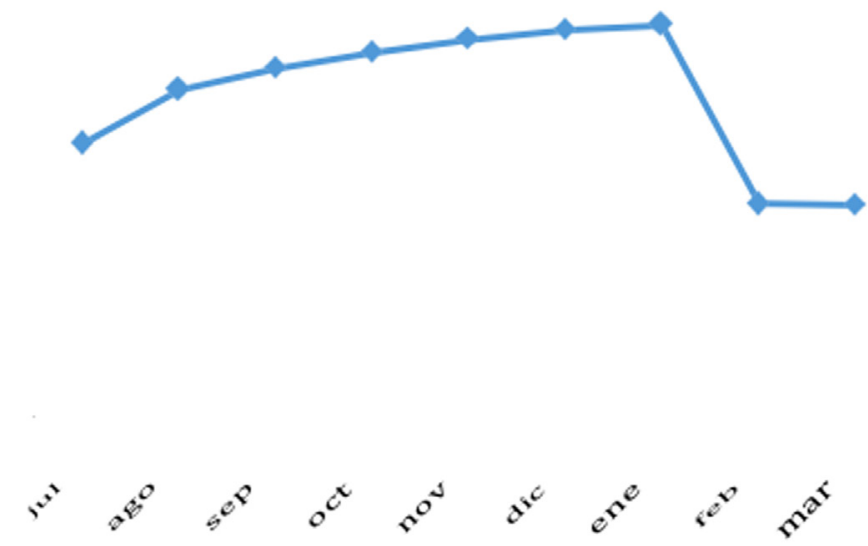

Nota: Elaboración propia a partir de los datos del ANSES

El escenario educativo que se plantea en el caso de Uruguay no difiere mucho de la problemática que afronta Argentina. El núcleo gordiano de la desigualdad se focaliza de nuevo, en la educación media que presenta desafíos importantes en relación mayoritariamente al tránsito entre cursos, al desempeño académico y a la culminación de niveles.

En el último informe publicado por el MIDE (2018) se pone de manifiesto que más del $60 \%$ de los jóvenes no logran terminar los estudios de educación media antes de los 20 ó 21 años, sólo el $6 \%$ de ellos los finaliza, seis años más tarde.

No obstante, el desarrollo que está realizando Uruguay de los programas monetarios en materia educativa no deja de ser paradigmático. A la etapa de reconocimiento internacional que ha tenido la línea de acción marcada en el «Programa de Aulas Comunitarias» (PAC) le ha seguido un periodo de abolición de muchas de las iniciativas de inclusión que se conformaron en el marco de un modelo de gobierno más social.

En la actualidad, el «Programa de Aulas Comunitarias» que se inició en 2007 ha dejado de ofertarse. Ha sido sustituido por el Programa: «Propuesta 2016» de acuerdo a motivos de carácter político. Las autoridades del Consejo de Educación Secundaria basándose en una interpretación interesada de las políticas de focalización consideraron la conveniencia de reemplazar espacios y profesionales de ámbitos no formales para centrar la atención (la inclusión) en los escenarios de intervención de marcos formales. 
Desde principios de responsabilidad compartida entre diferentes instituciones políticas (Ministerio de Desarrollo Social, Consejo de Educación Secundaria) y sociales (distintas Organizaciones no gubernamentales), el programa de aulas comunitarias ha estado dirigido a jóvenes entre 13 y 17 años en riesgo de exclusión social. Son adolescentes que abandonaron el sistema educativo, que no cursaron matriculación en el segundo ciclo y que cursando este ciclo tienen una situación muy comprometida. EI PAC se organizaba en distintas modalidades: A (primer año del ciclo), B (para alumnos desvinculados), C (egresados del PAC que cursan $2^{\circ}$ año en liceos) y Acompañamiento al egreso. Precisamente, la posibilidad de establecer cauces de comunicación y espacios de trabajo compartido entre instituciones muy diferentes constituye una de las claves para entender el éxito que ha llegado a convertir este programa en referente de las políticas de intervención en educación inclusiva (Mancebo y Monteiro, 2009).

Tabla 1. Características programas de inclusión seleccionados.

\begin{tabular}{|c|c|}
\hline \multicolumn{2}{|c|}{ PAÍSES, PROGRAMAS DE INCLUSIÓN SELECCCIONADOS Y CARACTERÍSTICAS } \\
\hline ARGENTINA & URUGUAY \\
\hline PROGRESAR & PROGRAMA DE AULAS COMUNITARIAS \\
\hline \multicolumn{2}{|c|}{ TIPO DE POLÍTICA SEGUIDA EN EL PROGRAMA } \\
\hline Focalizada & Focalizada \\
\hline \multicolumn{2}{|c|}{ COLECTIVOS A LOS QUE ESTÁ DIRIGIDO } \\
\hline $\begin{array}{l}\text { Jóvenes entre } 18 \text { y } 24 \text { años. } \\
\text { Vulnerabilidad.. }\end{array}$ & $\begin{array}{l}\text { Adolescentes entre } 13 \text { y } 17 \text { años. } \\
\text { Situación de riesgo social. }\end{array}$ \\
\hline \multicolumn{2}{|c|}{ OBJETIVOS QUE PERSIGUEN } \\
\hline $\begin{array}{l}\text { Generar equidad a través de la inclusión. } \\
\text { Ofrecer oportunidades de protección social y de } \\
\text { construcción de un proyecto profesional. } \\
\text { Combatir la exclusión a través de } \\
\text { oportunidades educativas. }\end{array}$ & $\begin{array}{l}\text { Generar equidad a través de la inclusión. } \\
\text { Crear a los docentes y profesionales espacios compartidos } \\
\text { de preocupación con relación al desarrollo escolar y } \\
\text { personal de los estudiantes. } \\
\text { Implicar a un mayor número de instituciones y } \\
\text { de profesionales. }\end{array}$ \\
\hline \multicolumn{2}{|c|}{ PRINCIPALES DIFICULTADES } \\
\hline
\end{tabular}

Nota: Elaboración propia.

\section{LA PRIVATIZACIÓN COMO RESPUESTA POLÍTICA A LA INCLUSIÓN}

En su intento por introducir racionalidad en los modos de mercado educativos, Ball $(2008,2012)$ realiza una clasificación de los tipos de privatización existentes, diferenciando entre otras, formas endógenas y exógenas. En estos últimos años la permeabilidad que han tenido las estructuras económicas en la organización y el funcionamiento de los centros escolares han ido configurando un sistema educativo cada vez más próximo a la cultura de mercado.

Las políticas de inclusión no representan ninguna excepción, universalizar la educación o implementar actuaciones de lucha contra el abandono escolar son declaraciones gubernamentales que forman parte de un lenguaje utilitario que no se traduce en formas y estrategias más democráticas.

Tampoco están ajenos de estas nuevas tendencias países con contextos económicos complejos, como es el caso de Argentina y gobiernos que se han cimentado con mecanismos más sociales, como ocurre con Uruguay.

Verger, Fontdevilla y Zancajo (2016) y Verger, Moschetti y Fontdevilla (2017), en su trabajo posterior sobre la privatización de la educación en América Latina alertan de las nuevas formas de desmantelar 
las políticas públicas que está empleando Argentina en la gestión de la educación, como son: la contratación externa de servicios periféricos o directamente de escuelas, los formatos que se introducen a partir de las alianzas público-privadas, las reformas de cuasi-mercado, y los incentivos financieros o salariales, entre otras, y coinciden con el análisis realizado en el Informe sobre la Campaña Argentina por el Derecho a la Educación (2018) en cuanto a la novedad de las formas que se emplean: se mantienen las relaciones entre sectores, pero se introduce el componente de la filantropía que se vincula a la calidad (caso de la organización Enseñá x Argentina que forma parte de una red global) y se crean nuevas filiaciones institucionales para el tratamiento y la difusión de la información pública.

Figura 3. Algunos indicadores del Mapa de la privatización en Uruguay y Argentina.

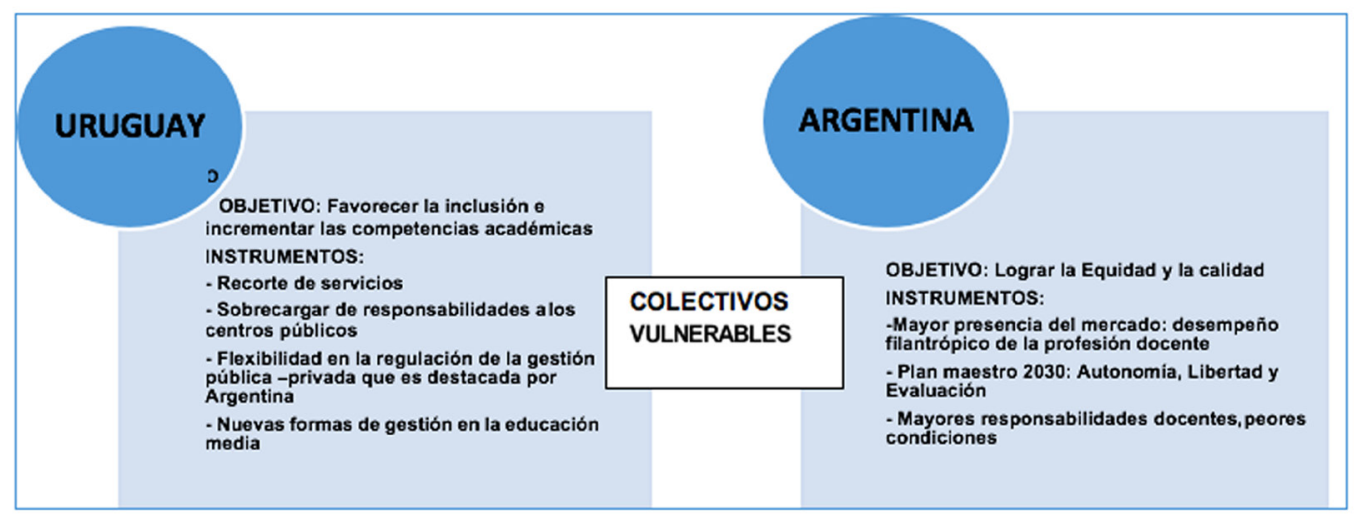

Nota: Elaboración propia.

Básicamente, los principios sobre los que se construye la privatización, tal y como se recoge en la figura 3, siguen manteniendo un interés por administrar servicios públicos con formas privadas y por regular las organizaciones con reglas de mercado, sin embargo, se modifican las formas y se amplían los grupos de interés. Las problemáticas que con anterioridad eran asociadas con los poderes públicos comienzan a ser asumidas como responsabilidades del sector privado. Hay una mayor preocupación por mostrar una sensibilidad hacia colectivos en situación de vulnerabilidad y hay también un interés por asimilar las actuaciones a la filantropía (Au y Lubienski, 2016; Saura, 2016).

Bordoli, et al (2017) denuncian en el caso de Uruguay una línea orientada a la desvalorización de los servicios públicos que en el desarrollo y el estudio que hemos hecho del Programa PAU reflejaría además un desplazamiento de responsabilidades a las instituciones públicas que antes eran compartidas entre la gestión pública y la social. Lo que puede estar dirigido a un desprestigio a través de la saturación de responsabilidades y la desatención en su desarrollo.

\section{DISCUSIÓN Y CONCLUSIONES}

A lo largo de este trabajo se ha puesto de manifiesto la progresiva representatividad que están teniendo las políticas educativas de inclusión en el marco de la agenda 2030. Su vinculación con las preocupaciones institucionales se consolida a través de enfoques estratégicos que le estarían otorgando un carácter instrumental, reflejado en la implementación que hacen los gobiernos en materia de educación inclusiva.

En este sentido, consideramos que son dos los mecanismos sobre los que se estaría orientando la inclusión en el contexto argentino y en el escenario uruguayo. Por una parte, hablamos de un componente de carácter económico: las transferencias monetarias, que gozan de un amplio (y controvertido) recorrido en América latina y cuya presencia en los países de referencia se ha ido consolidando desde criterios de financiación. De este modo, el trabajo muestra el incremento producido en políticas de transferencias económicas, aunque, contrariamente a lo que se proyecta en estos programas, nos ha sido difícil establecer una reciprocidad en el plano educativo, en tanto que las transferencias abren un camino hacia la dimensión asistencial que poco o nada tiene que ver con el desarrollo del derecho a la educación, bien delimitado en otros marcos de gobierno. 
Por otra parte, esta vertiente más subsidiaria de la protección estaría en línea con nuevas tendencias en la privatización, que constituye el segundo mecanismo sobre el que se estaría sustentando la inclusión educativa en Argentina y paradójicamente también en Uruguay, a pesar de la tradición democrática y social que durante mucho tiempo ha acompañado a este país.

En este punto preocupan los desarrollos que se están haciendo de los servicios públicos y no nos pueden dejar indiferentes ni el uso utilitarista de la inclusión como un objeto de mercado, ni tampoco los discursos de oportunidades y las formas de voluntarismo privado-público con el que se ejerce la equidad, acciones que romperían con cualquier posibilidad de institucionalizar los derechos relativos a la educación.

\section{REFERENCIAS BIBLIOGRÁFICAS}

Au, W. y Lubienski, C. (2016). The role of the Gates Foundation and the philanthropic sector in shaping the emerging education market: Lessons from the US on privatization of schools and education governance. In: Verger, A, Lubienski, C, Steiner-Khamsi, G (eds) The Global Education Industry. Routledge, (pp. 27-43).

Ball, S.J. y Youdell, D. (2008). Hidden privatisation in public education. Busseles: Education Internacional.

Ball, S. J. (2012). Global Education Inc. New Policy Networks and the Neo-liberal Imaginary. New York: Routledge.

Baraibar, X. (2011). Más Que Algo A Menos Que La Nada: Transferencias Condicionas y educación. SER Social, 13 (29), 195-217.

Bogliaccini, J.A. (2018). La educación en Uruguay mirada desde los objetivos de desarrollo sostenible. Montevideo (Uruguay): INEEd y Unicef.

Bordoli, E., Martinis, P., Moschetti, M., Conde, S. y Alfonzo M. (2017). Privatización Educativa en Uruguay: políticas, actores y posiciones. Internacional de la Educación.

Campaña Argentina por el Derecho a la Educación CADE (2018). Aportes a las políticas públicas educativas. Buenos Aires: Recuperado de https://drive.google.com/file/d/1IOIRsadDD9YPhMDFS95p7eFtULO4JJOU/view.

Cecchini, S. y Atuesta, B. (2017). Programas de transferencias condicionadas en Ámérica Latina y el Caribe. Tendencias de Cobertura e inversión. CEPAL - Serie Políticas Sociales, 224. Santiago: Naciones Unidas.

Cirmi, L. (2017). ¿Qué está pasando con el Progresar? Artículo de opinión en Infobae. Recuperado de https://www.infobae. com/opinion/2017/06/30/que-esta-pasando-con-el-progresar/.

Hornes, M. (2014). Transferencias condicionadas y sentidos plurales: el dinero estatal en la economía de los hogares argentinos, Antipoda, 18, 61-83.

Ibarrarán, P. y Cueva. (2017). Condicionalidades para el desarrollo de capacidades. En P. Ibarrarán, N. Medellín, F. Regalia y M. Stampini (Ed.) Así funcionan las transferencias condicionadas. Buenas prácticas a 20 años de implementación (pp.34-54) BID (Banco Interamericano de Desarrollo).

Ibarrarán, P., Regalia, N., y Stampini, M. (2016). Así funcionan las transferencias condicionadas. Buenas prácticas a 20 años de implementación. BID (Banco Interamericano de Desarrollo).

Mancebo, M. ${ }^{a}$ E. y Monteiro, L (2009). El Programa de Aulas Comunitarias de Uruguay. Un Puente hacia la inclusión en la Educación Media. REICE Revista Iberoamericana sobre Calidad, Eficacia y Cambio en Educación, 7 (4), 277-291.

Martens (2017). Políticas públicas para los ODS. Spotlight. Enfoques sobre Desarrollo Sostenible 2017. Informe del Grupo de Reflexión de la Sociedad Civil sobre la Agenda 2030 para el Desarrollo Sostenible (pp. 11-19). Recuperado de http://www.2030spotlight.org.

MIDES (2018). Reporte Uruguay 2017. Uruguay: Ministerio de Desarrollo Social. Recuperado de https://www.opp.gub.uy/ images/ReporteUruguay2017.pdf.

Naya, L.M. y Dávila, P. (2017). El derecho a la educación y la educación en el mundo. En Caride, J.A., Vila, E.S. y Martín V.M. (Coord.). Del derecho a la educación a la educación como derecho. (pp.13-39) Granada: GEU.

Saura, G. (2016). Neoliberalización filantrópica y nuevas formas de privatización educativa: La red global Teach For All en España. RASE Revista de Sociología de la Educación, 9 (2), 248-264.

Ramos, J.M. (2016). Las entidades de la frontera norte y la Agenda 2030: retos para una gobernanza para el desarrollo. Secuencia, (98), 228-256. Recuperado de https://dx.doi.org/10.18234/secuencia.v0i98.1388. 
UNESCO (2015). Declaración de Incheon. Educación 2030: Hacia una educación inclusiva y equitativa de calidad y un aprendizaje a lo largo de la vida para todos. Foro Mundial de Educación. http:// unesdoc.unesco.org/images/0023/002331/233137S.pdf.

Verger, A. Moschetti, M. y Fontdevilla, C. (2017). La privatización educativa en América Latina. Una cartografía de políticas, tendencias y trayectorias. Education Internacional. Recuperado de https://download.ei-ie.org/Docs/WebDepot/Privatizacion\%201-Abril.pdf.

Verger, A. Fontdevilla, C. y Zancajo, A. (2016).The Privatization of Education: A Political Economy of Global Education Reformby. New York: Teachers College Press.

Westheimer, J. (2015). What Kind of Citizen? Educating Our Children for the Common Good. Nueva York: Teachers College Press.

Wulff, A. (2017). Lucrar con el ODS 4. Spotlight. Enfoques sobre Desarrollo Sostenible 2017. Informe del Grupo de Reflexión de la Sociedad Civil sobre la Agenda 2030 para el Desarrollo Sostenible (pp.60-66). Recuperado de http:// www.2030spotlight.org. 